\title{
Sistematização de uma sessão de treinamento especifico para surfistas
}

\author{
Krom Marsili Guedes ${ }^{\mathrm{ab}}$, Pedro Souza ${ }^{\mathrm{b}}$, Rodrigo Pereira da Silva ${ }^{\mathrm{abc}}$, Dilmar Pinto Guedes Jr ${ }^{\text {ade }}$ \\ aUniversidade Metropolitana de Santos (UNIMES), Santos-SP. \\ bUniversidade Federal de São Paulo (UNIFESP), Santos-SP. \\ 'Faculdade Praia Grande (FPG), Praia Grande-SP. \\ dUniversidade Santa Cecilia (UNISANTA), Santos - SP. \\ eCentro de Estudos de Fisiologia do Exercício e Treinamento (CEFIT), São Paulo-SP.
}

RESUMO Nas últimas décadas o surf cresceu de forma significativa no Brasil. Atualmente são mais de 3 milhões de praticantes, tornando o nosso país o líder com o maior número de adeptos no mundo. Esses dados já justificam a importância de sugerir um modelo de sessão de treinamento físico específico para a modalidade surf, com o objetivo de melhorar o rendimento dos surfistas, além de prevenir o risco potencial de algumas lesões. O surf, como esporte, é classificado como uma modalidade complexa de coordenação. A sua prática exige o treinamento e desenvolvimento de diferentes capacidades e habilidades físicas. A sessão de treinamento proposta no presente estudo é uma alternativa de aula que pode ser utilizada para o público praticante de surf em geral. No entanto, um surfista competitivo pode, em algum momento de sua periodização do treinamento, utilizar tal modelo de sessão, considerando a individualização, o volume e a intensidade da carga.

PALAVRAS-CHAVE: preparação fisica; surf; periodização.

Aceito 2 de março de 2021 Publicado online 26 de abril de 2021

Cite este artigo: Guedes et al. (2021) Sistematização de uma sessão de treinamento especifico para surfistas. Multidisciplinary Science Journal 3: e2021008, doi: 10.29327/multiscience.2021008.

\section{Systematization of a specific training session for surfers}

ABSTRACT In the last decades, the surf has grown significantly in Brazil. There are currently more than 3 million practitioners, making our country the leader with the largest number of supporters in the world. These data already justify the importance of suggesting a specific physical training session model for the surf modality, with the objective of improving the performance of surfers, in addition to preventing the potential risk of some injuries. Surfing, as a sport, is classified as a complex form of coordination. Its practice requires the training and development of different physical skills and abilities. The training session proposed in the present study is a class alternative that can be used for the surfing public in general. However, a competitive surfer may, at some point during his training period, use this session model, considering the individualization, the volume and the intensity of the load.

KEYWORDS: physical preparation; surf; periodization.

\section{Introdução}

O surf foi incluído no cronograma olímpico no ano de 2016, dando inicio a uma nova era no universo competitivo da modalidade (ISA 2016). Complexos esportivos com diferentes equipamentos estarão disponíveis para que surfistas e treinadores desenvolvam a performance esportiva da melhor maneira possível. Contudo, em um primeiro momento, a grande maioria dos estudos existentes na literatura eram voltados para a melhora da performance na remada do surfista. Remar é extremamente importante, porém o surfista mais rápido na remada não necessariamente é o de melhor performance na onda (Guedes et al 2018). Atualmente, diversos estudos relatam a importância do treinamento 
de força e potência muscular no surf (Tran et al 2015; Gamboa et al 2017; Gravestock et al 2017; Secomb et al 2017; Forsyth et al 2020). A literatura atual carece de intervenções com diferentes modelos de treinamento planejados para as exigências físicas especificas exigidas pelo surf.

Ainda que o surf possa ser considerado um esporte de contemplação e lazer, tendo o estilo de vida proporcionado pela prática como pano de fundo, muitos adeptos buscam uma auto superação ao se desafiarem no ambiente imprevisível do mar em ondas de diferentes proporções (Souza 2013). Surfar é uma atividade complexa que requer do surfista as manifestações de força máxima, forca explosiva (potência) e forca de resistência dos membros inferiores e superiores, resistência aeróbia, equilíbrio dinâmico, velocidade de reação e ação, flexibilidade e mobilidade (Tran et al 2015; Mendez-Villanueva et al 2006). De acordo com Chantel e Brown (2016) o surf é caracterizado por gestos intermitentes de alta intensidade, que exigem essas características para que o surfista execute as manobras, como os aéreos, as batidas, os floaters, da forma mais radical e eficiente possível. A execução de exercícios que reproduzam os movimentos específicos do esporte fora da água pode auxiliar no treinamento da técnica do movimento dentro da água, por isso devem ser inseridas nos programas de treinamento para surfistas em conjunto com os exercícios de força e potência tradicionais (Forsyth et al 2020; Chantel et al 2016).

Apesar do aumento das pesquisas cientificas abrangendo estratégias para a melhora da performance do surfista, a literatura não apresenta ainda um modelo de treinamento específico para a modalidade. $O$ presente estudo apresenta um modelo de sessão de treinamento físico específico para a modalidade surf (Figura 1), com o objetivo de melhorar o rendimento dos surfistas, além de prevenir o risco potencial de algumas lesões.

\section{Objetivo da sessão de treinamento: Adaptação multi-sistêmica}

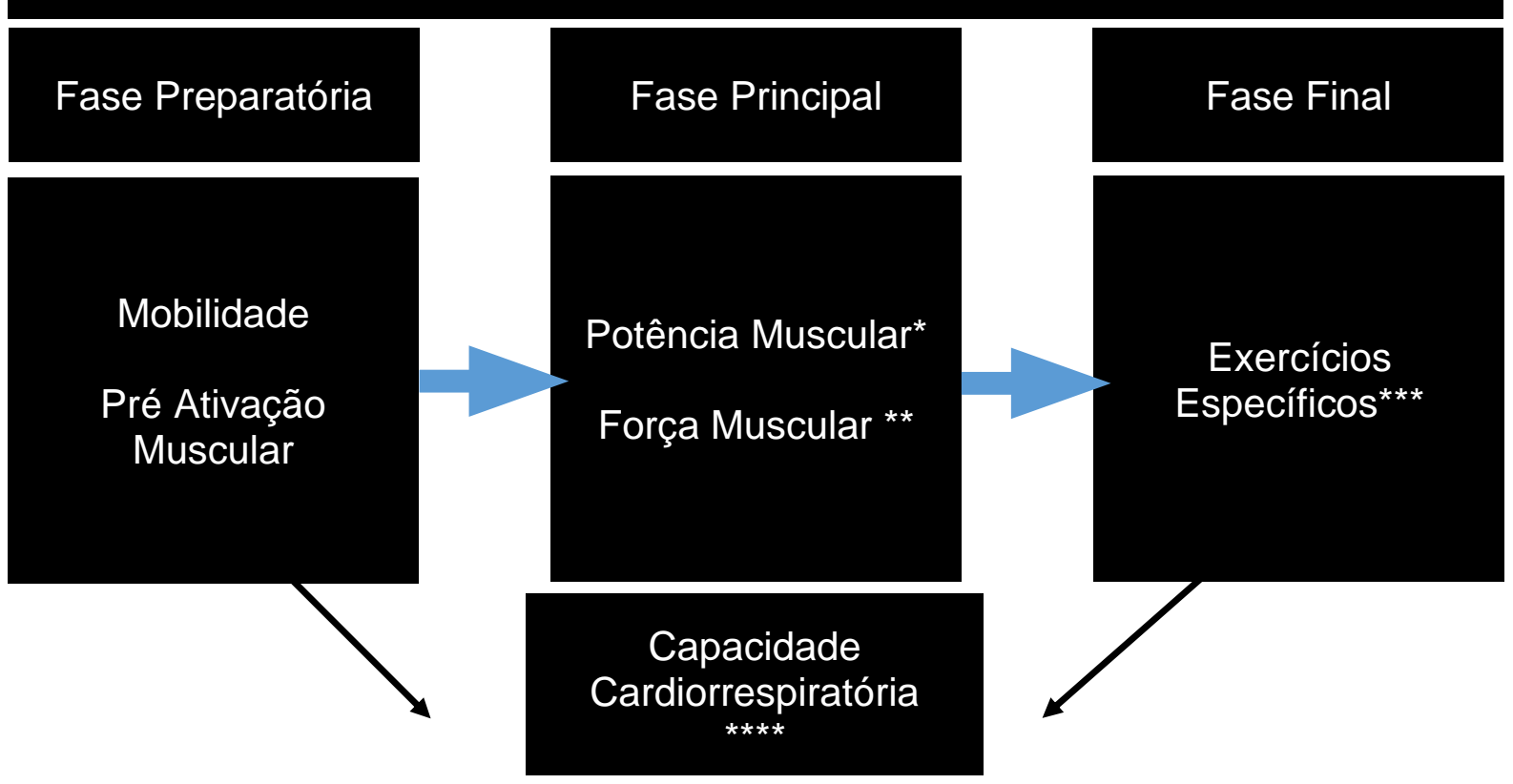

* O trabalho de potência muscular é realizado alternado por segmento em progressão horizontal, intervalos de 3 minutos são adotados para recuperação da via ATP-CP.

** A força muscular segue o mesmo padrão de trabalho, com intervalos de 3 minutos, em algumas sessões e as cargas complexas podem ser adotadas, um exercício de potência muscular seguido de um exercício de força muscular, mantendo o mesmo tempo de intervalo de 3 minutos.

*** Os exercícios específicos são realizados em forma de circuito, com pausas ativas em algum ergômetro para simular a volta do surfista ao "outside".

**** A capacidade cardiorrespiratória, considerada condicionante para o surf, é treinada no contexto da sessão de treinamento.

Figura 1 Modelo de uma sessão de Treinamento Específico para surfistas. 


\section{Fase Preparatória}

Na fase preparatória da sessão o organismo deve passar do estado de repouso relativo para o estado de "trabalho", permitindo com que as tarefas da fase principal sejam resolvidas de maneira eficiente (Gomes 2009). De acordo com Resende-Neto e Silva-Grigoletto (2020), dentro de uma sessão de treinamento funcional a preparação para o movimento tem como função garantir o máximo de proveito dos estímulos e assegurar a integridade física dos participantes na realização de movimentos mais complexos. A mobilidade articular, descrita em estudos que avaliam a amplitude das principais articulações envolvidas na prática do surf, revelam que ao tentar realizar manobras complexas, são exigidas altas taxas de produção de força em diversas amplitudes articulares (Lundgren et al 2015). No trabalho de Lundgren et al (2013) também alerta que limitações nas articulações dos tornozelos podem inibir o desempenho. Praticantes de nível mais elevado, capazes de executar manobras como floater e aéreos devem preocupar-se com treinos de estabilização articular, para reduzir danos provenientes dos impactos durante a aterrisagem dessas manobras (Tran et al 2015; Forsyth et al 2014; Forsyth et al 2019).

O treinamento do core deve fazer parte da rotina de treinos, uma vez que contribui significativamente para otimização do desempenho de surfistas e tem um importante papel na prevenção de lesão (Forsyth et al 2020; Axel et al 2018; Silva et al 2018). Além disso, estudos recentes demonstram que ter uma boa estabilidade de tronco é fundamental para a realização das tarefas funcionais do surf (Silva et al 2018). O exercício realizado em superfície instável não estimula satisfatoriamente o desenvolvimento da força e da potência de membros inferiores, merecendo cautela na sua aplicação em surfistas (Tran et al 2015; Bernards et al 2017). No entanto, Mendez-Villanueva e Bishop (2005) lembram que o surf é praticado em ambiente instável e imprevisível, o que sugere que os exercícios criados e aplicados nas superfícies instáveis deveriam ser utilizados dentro de uma proposta de treinamento altamente específico, constituído da reprodução do gesto esportivo, de modo que se alie o treinamento físico ao técnico em momentos oportunos da preparação (Moreira e Peixoto 2014; Secomb et al 2017; Axel et al 2018; Forsyth et al 2019; Forsyth et al 2020). Em um momento de preparação para o movimento os exercícios nas superfícies instáveis podem colaborar para uma estimulação do sistema nervoso central e pré ativação muscular, exercendo não um papel principal no programa de treinamento, mas sim como elemento potencializador do resultado final.

\section{Fase Principal}

Na fase principal da sessão de treinamento devem ser resolvidas as principais tarefas (Gomes 2009). Na presente sessão de treinamento a fase principal objetiva o aumento da força e da potência muscular. Algumas pesquisas demonstraram que em âmbito competitivo, atletas bem ranqueados apresentam melhor desempenho em testes de salto vertical e força isométrica, além de apresentarem maiores taxas de massa magra (Tran et al 2015; Gamboa et al 2017). Manobras realizadas por surfistas mais pesados podem impressionar os juizes e telespectadores pela quantidade de água jogada para cima da onda. Surfistas que buscam surfar com maior intensidade em suas manobras devem realizar exercícios de caráter neuromuscular em suas sessões de treinamento (Gravestock et al 2017; Secomb et al 2017). Exercícios denominados "básicos" da musculação com objetivo de desenvolver a força muscular são importantes, assim como exercícios como saltos pliométricos para que o atleta possa desenvolver o máximo de força em um curto período de tempo, possibilitando a realização de manobras explosivas com maior frequência e intensidade (Bernards et al 2017).

Os exercícios de salto podem constar de saltos simples em uma fase inicial e progredir para saltos com rotações, buscando atender ao gesto motor da maioria das manobras do surf. A capacidade de mudança de direção em alta velocidade durante a realização das manobras também está relacionada com a competência de rotacionar o tronco em grandes amplitudes (Furness et al 2015). A força isoinercial do tronco é importante para que o surfista mude a direção durante um movimento em alta velocidade, frenagem e mudança para direção oposta (Suchomel et al ,2018, Chaabene, et al 2018). Essa tarefa está presente no momento em que o surfista realiza uma cavada e entra na manobra olhando para a face da onda e posteriormente gira seu tronco no sentido da praia para finalizar uma manobra.

\section{Fase Final}


É fundamental que exista uma transferencia da melhora da aptidão física geral para a performance do surfista na onda (Bernards et al 2017). Dessa forma, os exercícios da sessão devem respeitar o princípio da especificidade. As manobras são ações técnicas que expressam o desempenho (Moreira e Peixoto 2014) São realizadas na parede da onda e os surfistas se destacam a medida que alcançam cada vez mais radicalidade e complexidade na sua construção (Peirão e Dos Santos 2012; Sheppard et al 2013; Forsyth et al 2017). Em média, os surfistas realizam 4 a 5 manobras numa onda, percorrem uma distância entre 15 e 150 metros e o tempo de duração desse percurso varia entre 4 e 27 segundos.

O surf apresenta algumas particularidades que o diferencia de todos os outros esportes; uma onda nunca é igual a outra e existem momentos onde simplesmente não tem ondas, dificultando o treinamento especifico dos atletas. De acordo com Forsyth et al, (2020), 100\% do padrão técnico da manobra Front Side Air e 60\% do Front Side air reverse pôde ser desempenhado em uma simulação fora d'agua, informação importante para correções de padrões técnicos de braços e pernas durante a manobra. No entanto, os autores ressaltam a importância do treinador em promover uma analise do movimento e um feedback correto para os atletas.

Outras manobras como rasgadas e batidas também podem ser reproduzidas fora d'agua e introduzidas em alguns momentos em programas e sessões de treinamento. De fato, o aumento do salto vertical dos surfistas está correlacionado com os resultados dos surfistas mais bem ranqueados (Tran et al 2014; Gamboa et al 2017), porém é preciso transferir essa potência adquirida no salto para o gesto técnico da manobra. $O$ objetivo do surfista é reproduzir o gesto da manobra com a maior força possível em um menor período de tempo e não saltar em cima da prancha. 0 bloco 3 da sessão de treinamento é a parte especifica do esporte, onde o objetivo é reproduzir com ajuda de equipamentos como bolas, elásticos e slides, além do próprio gesto corporal exercícios educativos para a aprendizagem do gesto técnico das manobras do surf. Como já comentado no texto o uso da instabilidade como parte da preparação especifica sessão do treinamento se justifica pelo esporte ser praticado em meio liquido, com condições imprevisíveis, gerando instabilidade (Mendez-Villanueva e Bishop 2005).

\section{Cardiorrespiratório}

Segundo Mendez-Villanueva e Bishop (2005), o surf é um esporte de atividade intermitente, em que se alternam momentos de remada de baixa e alta intensidade e percurso na onda com a realização de manobras. As remadas compõem cerca de $60 \%$ das atividades de uma sessão de surf, enquanto os períodos de 20 segundos em média consistem nessas ações realizadas em alta intensidade (Secomb et al 2015). Dessa maneira, se faz necessária a escolha de exercícios que atinjam os sistemas de energia utilizados na prática do surf, que segundo Farley et al (2016) pode ser constituído por treinamentos intervalados de alta intensidade. Por sua vez, quando combinados com exercícios que promovam melhora da capacidade aeróbia, geram adaptações que contribuem positivamente sobre os processos de recuperação Farley et al (2012). Além disso, exercícios de força direcionados aos membros superiores, principalmente em indivíduos com menos experiência no contexto do treino de hipertrofia, impactam diretamente na capacidade de remar em alta intensidade com aumento da velocidade e da resistência na prática dessa tarefa (Coyne et al 2017). De fato, fica evidente a exigência do aparelho cardiorrespiratório frente às demandas impostas aos sistemas aeróbio e anaeróbio, sobretudo por ações representadas por remadas de baixa e alta intensidade que incluem a maior parte das ações físicas de uma sessão de surf (Secomb et al 2015).

Dentro da proposta demonstrada, o treino segue utilizando o método em "circuito", onde pausas ativas podem ser adotadas para simular a remada do surfista através de exercícios cíclicos como esteira, bicicleta, remador e se possível, em um ambiente de treinamento considerado perfeito as pausas seriam realizadas com remadas de baixa intensidade no mar ou em uma piscina.

\section{Treinamento para o alto rendimento}

A periodização do treinamento consiste em criar um sistema de planos para distintos períodos que perseguem um conjunto de objetivos vinculados, sendo que o plano de treinamento prevê perspectivas a curto, médio e longo prazo (Gomes 2009). A periodização em blocos proposta pelo russo Yuri Verkoshanski propõe a distribuição da carga de forma concentrada em blocos ao longo do ciclo anual de treinamento (Gomes 2009). De acordo com Bernards et al (2017), a periodização em blocos demonstra-se eficiente para o surf por proporcionar o desenvolvimento da força e 
potência muscular, além de velocidade, capacidades fundamentais para a execução de manobras explosivas, além de minimizar a fadiga acumulada. De fato, o atleta de alto rendimento deve ser tratado de maneira diferente de um surfista recreativo.

\section{Considerações Finais}

O surf, como esporte, é classificado como uma modalidade complexa de coordenação. A sua prática exige o treinamento e desenvolvimento de diferentes capacidadaes e habilidades físicas. A sessão de treinamento proposta no presente estudo é uma alternativa de aula que pode ser utilizada para o público praticante de surf em geral. No entanto, um surfista competitivo pode, em algum momento de sua periodização do treinamento, utilizar tal modelo de sessão, considerando a individualização, o volume e a intensidade da carga. Além disso, o planejamento da temporada de treinamento vinculado ao calendário competitivo é fundamental para que o pico do desempenho seja alcançado no momento adequado. São necessários mais estudos considerando diferentes modelos de sessão, assim como a estruturação e planejamento em longo prazo para que se possa desenvolver um modelo específico de treinamento para surfistas recreacionais e competidores.

\section{Declaração de conflito de interesses}

Não há conflito de interesses entre os autores.

\section{Referências}

Anthony CC, Brown LE (2016) Resistance training considerations for female surfers. Strength \& Conditioning Journal 38:64-69.

Axel TA, Crussemeyer JA, Dean K, Young DE (2018) Field test performance of junior competitive surf athletes following a core strength training program. International journal of exercise science 11:696.

Bernards J, Blaisdell R, Light TJ, Stone MH (2017) Prescribing an annual plan for the competitive surf athlete: Optimal methods and barriers to implementation. Strength \& Conditioning Journal 39:36-45.

Chaabene H, Prieske O, Negra Y, Granacher U (2018) Change of direction speed: Toward a strength training approach with accentuated eccentric muscle actions. Sports Medicine 48:1773-1779.

Coyne JO, Tran TT, Secomb JL, Lundgren LE, Farley OR, Newton RU, Sheppard JM (2017) Maximal strength training improves surfboard sprint and endurance paddling performance in competitive and recreational surfers. The Journal of Strength \& Conditioning Research 31:244-253.

Da Resende-Neto AG, Da Silva-Grigoletto ME (2020) Systematization of Functional Training Sessions to Benefit Physical Fitness for Daily Activities in Older People. Int J Sports Exerc Med 6:157.

Farley OR, Abbiss CR, Sheppard JM (2016) Testing protocols for profiling of surfers' anaerobic and aerobic fitness: A review. Strength and Conditioning Journal 38:52-65.

Farley O, Harris NK, Kilding AE (2012) Anaerobic and aerobic fitness profiling of competitive surfers. The Journal of Strength \& Conditioning Research 26:2243-2248.

Forsyth JR, De La Harpe R, Riddiford-Harland DL, Whitting JW, Steele JR (2017) Analysis of Scoring of Maneuvers Performed in Elite Men's Professional Surfing Competitions. International journal of sports physiology and performance 12:1243-1248.

Forsyth JR, Riddiford-Harland DL, Whitting JW, Sheppard JM, Steele JR (2020) Training for success: Do simulated aerial landings replicate successful aerial landings performed in the ocean?. Scandinavian journal of medicine \& science in sports 30: 878-884.

Forsyth JR, Steele JR (2019) Which drop height is most suitable to simulate landing a floater? n. December 2019.

Forsyth J, Riddiford-Harland DL, Lundgren L, Whitting JW, Sheppard JM, Steele JR (2014) The influence of ankle range of motion on lower limb muscle activity when landing an aerial manoeuvre in surfing. 9th Australasian Biomechanics Conference.

Furness J, Hing WA, Pope RR, Climstein M (2015) Trunk mobility in the sagittal and horizontal planes: clinical methods to quantify movement in an elite surfing population. In CONNECT 2015 Physiotherapy Conference.

Gamboa I, Yanci J, Granados C, Camara J (2017) Comparison of anthropometry and lower limb power qualities according to different levels and ranking position of competitive surfers. The Journal of Strength \& Conditioning Research 31:2231-2237. 
Gravestock HJ, Barlow MJ (2017) The Use of Tensiomyography To Evaluate Neuromuscular Profile and Lateral Symmetry in Competitive. Advances in Skeletal Muscle Function Assessment 1:15-20.

Gomes AC (2009) Treinamento desportivo: estrututuração e periodização. Artmed Editora.

Guedes KM, da Silva RP, Dourado VZ, Junior DPG (2018) Perfil e aspectos metodológicos da preparação física de surfistas. Revista Brasileira de Fisiologia do Exercício 17:234-246.

ISA (2016) International surfing association adds 97 th member as drive for global growth continues: International Surfing Association. Disponível em: http://www.isasurf.org/international-surfing-association-adds-97th-member-as-drive- forglobal-growth-continues/ 2015.

Lundgren LE, Tran TT, Nimphius S, Raymond E, Secomb JL, Farley OR, Sheppard JM (2015) Development and evaluation of a simple, multifactorial model based on landing performance to indicate injury risk in surfing athletes. International journal of sports physiology and performance 10:1029-1035.

Lundgren L, Tran T, Farley O, Secomb J, Nimphius S, Newton R, Sheppard JM (2013) Ankle range of motion among surfing athletes. Australian Strength and Conditioning Association 21:121-124.

Mendez-Villanueva A, Bishop D (2005) Physiological aspects of surfboard riding performance. Sports Medicine 35:55-70.

Mendez-Villanueva A, Bishop D, Hamer P (2006) Activity profile of world-class professional surfers during competition: Acase study. The Journal of Strength \& Conditioning Research 20:477-482.

Moreira M, Peixoto C (2014) Qualitative task analysis to enhance sports characterization: a surfing case study. Journal of human kinetics 42:245.

Peirão R, Santos SGD (2012) Critérios de julgamento em campeonatos internacionais de surfe profissional. Revista Brasileira de Cineantropometria \& Desempenho Humano 14:439-449.

Secomb JL, Farley OR, Nimphius S, Lundgren L, Tran TT, Sheppard JM (2017) The training-specific adaptations resulting from resistance training, gymnastics and plyometric training, and non-training in adolescent athletes. International Journal of Sports Science \& Coaching 12:762-773.

Secomb JL, Sheppard JM, Dascombe BJ (2015) Time-motion analysis of a 2-hour surfing training session. International journal of sports physiology and performance 10:17-22.

Sheppard JM, Nimphius S, Haff GG, Tran TT, Spiteri T, Brooks H, Newton RU (2013) Development of a comprehensive performancetesting protocol for competitive surfers. International Journal of Sports Physiology and Performance 8:490-495.

Silva B, Clemente FM, Martins FM (2017) Associations between functional movement screen scores and performance variables in surf athletes. The Journal of sports medicine and physical fitness 58:583-590.

Souza PC (2013) Surf: do desenvolvimento histórico ao profissionalismo. Acta Brasileira do Movimento Humano 3:84-98.

Suchomel TJ, Nimphius S, Bellon CR, Stone MH (2018) The importance of muscular strength: training considerations. Sports medicine 48:765-785.

Tran TT, Lundgren L, Secomb J, Farley OR, Haff GG, Seitz LB, Sheppard JM (2015) Comparison of physical capacities between nonselected and selected elite male competitive surfers for the national junior team. International journal of sports physiology and performance 10:178-182.

Tran TT, Nimphius S, Lundgren L, Secomb J, Farley OR, Haff GG, Sheppard JM (2015) Effects of unstable and stable resistance training on strength, power, and sensorimotor abilities in adolescent surfers. International Journal of Sports Science \& Coaching 10:899-910. 